\title{
THE MOTION OF A FLAT PLATE FROM REST IN A VISCO-ELASTIC LIQUID
}

\author{
by F. M. LESLIE \\ (Received 30th November 1962)
}

\section{Introduction}

Oldroyd (1) considered non-Newtonian liquids for which the stress tensor $s_{i k}$ and the rate of strain tensor $e_{i k}=\frac{1}{2}\left(v_{k, i}+v_{i, k}\right)$ are related as follows

$$
\begin{aligned}
s_{i k} & =p_{i k}-p g_{i k}, \ldots \ldots \ldots \ldots \ldots \ldots \ldots \ldots \ldots \ldots \ldots \ldots \\
p^{i k}+\lambda_{1} \frac{\delta}{\delta t} p^{i k}+\mu_{0} e^{i k} p_{j}^{j}+v_{1} e^{j l} p_{j l} g^{i k} & =2 \eta_{0}\left(e^{i k}+\lambda_{2} \frac{\delta}{\delta t} e^{i k}+v_{2} e^{j l} e_{j l} g^{i k}\right) .
\end{aligned}
$$

Here $v_{i}$ denotes the velocity vector, $p$ an isotropic pressure, $g_{i k}$ the metric tensor, and $t$ time; $\eta_{0}$ is a constant having the dimensions of viscosity and $\lambda_{1}, \lambda_{2}, \mu_{0}, v_{1}, v_{2}$ are constants having the dimension of time. Covariant suffixes are written below, contravariant above and the usual summation convention is employed. A suffix $i$ following a comma indicates covariant differentiation with respect to the space variable $x^{i}$. The derivative denoted by $\frac{\delta}{\delta t}$ is the " convected derivative" (2). For any second order contravariant tensor $B^{i k}$ we have

$$
\frac{\delta}{\delta t} B^{i k}=\frac{\partial}{\partial t} B^{i k}+v^{j} B_{,}^{i k}+w_{\cdot m}^{i} B^{m k}+w_{\cdot m}^{k} B^{i m}-e_{m}^{i} B^{m k}-e_{m}^{k} B^{i m},
$$

where $w_{i k}=\frac{1}{2}\left(v_{k, i}-v_{i, k}\right)$.

Provided that the five constants $\lambda_{1}, \lambda_{2}, \mu_{0}, v_{1}, v_{2}$ satisfy certain inequalities, Oldroyd showed that in theory liquids of the class defined by equations (1) and (2) exhibit for all rates of shear non-Newtonian flow properties which have been observed in polymer solutions. These liquids have a variable apparent viscosity in simple shearing, decreasing with increasing rate of strain from a value $\eta_{0}$ at low rates of strain to a value $\eta_{1}\left(<\eta_{0}\right)$ at high rates. They show the Weissenberg climbing effect and have a distribution of normal stresses associated with an extra tension along the streamlines in many types of simple flow, having an isotropic state of stress normal to the streamlines. Also for flow at low rates of shear such liquids are characterised by three constants, a coefficient of viscosity $\eta_{0}$, a relaxation time $\lambda_{1}$, and a retardation time $\lambda_{2}$. The author (3) showed that for a Stokes flow past a sphere the theoretical predictions are qualitatively in agreement with experiment. 
The aim of the present paper is to present some further solutions of equations (1) and (2) and the equations of conservation of mass and momentum for an incompressible liquid of density $\rho$,

$$
\left.\begin{array}{rl}
e_{i}^{i} & =0, \\
\frac{\partial v^{i}}{\partial t}+v^{k} v_{, k}^{i} & =\frac{1}{\rho} s_{, k}^{i k}
\end{array}\right\} .
$$

The problem considered is that of an infinite flat plate immersed in a liquid characterised by equations (1) and (2) and set in motion with a velocity $A t^{\frac{1}{2}}$ along a straight line in its own plane, where $A$ is a constant. Solutions as expansions in terms of time are obtained for both small and large time for the full equations. A solution of the equations in linearised form is obtained for all time by a Pohlhausen technique.

For a Newtonian fluid the drag on a flat plate whose velocity is $A t^{\frac{1}{2}}$ is a constant independent of time. For the non-Newtonian liquid considered the drag is initially different from this constant value by a factor $\left(\lambda_{2} / \lambda_{1}\right)^{\frac{1}{2}}$. Since $\lambda_{2}$ is less than $\lambda_{1}(4)$ this factor is less than unity and may be as small as one half (5). At large times $\left(>10 \lambda_{1}\right)$ the non-Newtonian drag is less than the corresponding Newtonian value, the difference being of order $A^{2} \lambda_{1}^{2} / v_{0}$, where $v_{0}$ is the kinematic viscosity $\eta_{0} \rho^{-1}$. The Pohlhausen solution estimates the drag for intermediate times and suggests that it quickly assumes its asymptotic value.

\section{The Equations of Motion}

Cartesian co-ordinates $x, y, z$ are chosen such that the flat plate occupies the plane $x=0$ and moves parallel to the $y$-axis. All tensor quantities are expressed in terms of their physical components referred to these co-ordinates. Let $u, v, w$ be the components of the velocity vector. Since the plate is infinite a solution of the form

$$
u=0, \quad v=v(x, \mathfrak{t}), \quad w=0
$$

is examined. From symmetry

$$
p_{x z}=p_{y z}=0
$$

and we seek a solution in which the other partial stresses $p_{i k}$ are functions of $x$ and $t$ only, all vanishing at large $x$; and $p$ is a function of $x$ and $t$ which tends to the constant pressure $P$ at large $x$. Equations (2), (3) become

$$
\begin{aligned}
& p_{x x}+\lambda_{1} \frac{\partial}{\partial t} p_{x x}+v_{1} \frac{\partial v}{\partial x} p_{x y}=v_{2} \eta_{0}\left(\frac{\partial v}{\partial x}\right)^{2}, \\
& p_{y y}+\lambda_{1} \frac{\partial}{\partial t} p_{y y}+\left(v_{1}-2 \lambda_{1}\right) \frac{\partial v}{\partial x} p_{x y}=\left(v_{2}-2 \lambda_{2}\right) \eta_{0}\left(\frac{\partial v}{\partial x}\right)^{2}, \\
& p_{z z}+\lambda_{1} \frac{\partial}{\partial t} p_{z z}+v_{1} \frac{\partial v}{\partial x} p_{x y}=v_{2} \eta_{0}\left(\frac{\partial v}{\partial x}\right)^{2}
\end{aligned}
$$




$$
\begin{aligned}
& p_{x y}+\lambda_{1} \frac{\partial}{\partial t} p_{x y}-\lambda_{1} \frac{\partial v}{\partial x} p_{x x}+\frac{1}{2} \mu_{0} \frac{\partial v}{\partial x}\left(p_{x x}+p_{y y}+p_{z z}\right)=\eta_{0}\left(\frac{\partial v}{\partial x}+\lambda_{2} \frac{\partial^{2} v}{\partial x \partial t}\right), \\
& \frac{\partial p_{x x}}{\partial x}=\frac{\partial p}{\partial x}, \quad \rho \frac{\partial v}{\partial t}=\frac{\partial p_{x y}}{\partial x}
\end{aligned}
$$

The boundary conditions are that there is no slip at the plate and that there is no disturbance initially or at large distance from the plate, i.e.

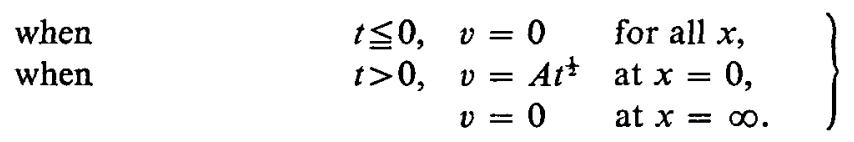

Since there is no disturbance initially it is assumed that in the fluid the stress is zero initially. This gives the following boundary conditions,

when

$$
t \leqq 0, \quad p_{x x}=p_{y y}=p_{z z}=p_{x y}=0 \text { for } x>0 .
$$

\section{Solution of the Equations}

To obtain solutions of the equations (4)-(8) for the boundary conditions (9), (10) a similarity variable $s=x / 2 t^{\frac{1}{2}}$ is introduced and $s, t$ are taken as independent variables.

For solutions for small time let

$$
\begin{aligned}
v & =t^{\frac{1}{2}} f_{0}(s)+t^{\frac{3}{2}} f_{1}(s)+\ldots, \\
p_{x y} & =g_{0}(s)+t g_{1}(s)+\ldots \\
p_{x x} & =p_{z z}=h_{0}(s)+t h_{1}(s)+\ldots, \\
p_{y y} & =k_{0}(s)+t k_{1}(s)+\ldots
\end{aligned}
$$

From equations (4)-(8),

$$
\left.\begin{array}{rl}
g_{0}^{\prime} & =\rho\left(f_{0}-s f_{0}^{\prime}\right), \\
2 g_{0}^{\prime} & =a^{2} \rho f_{0}^{\prime \prime}, \\
h_{0}^{\prime} & =k_{0}^{\prime}=0,
\end{array}\right\}
$$

where $a^{2}=v_{0} \lambda_{2} / \lambda_{1}$ and ' denotes differentiation with respect to the similarity variable $s$. The relevant boundary conditions are

$$
\begin{gathered}
f_{0}(0)=A, \quad f_{0}(\infty)=0, \\
g_{0}(\infty)=h_{0}(\infty)=k_{0}(\infty)=0 .
\end{gathered}
$$

The solution of equations (11) for those boundary conditions is

$$
\begin{aligned}
& f_{0}(s)=A\left(e^{-s^{2} / a^{2}}-2 s / a \operatorname{erfc}(s / a)\right), \\
& g_{0}(s)=-a \rho A \operatorname{erfc}(s / a), \\
& h_{0}(s)=k_{0}(s)=0,
\end{aligned}
$$


where

From equations (4)-(8)

$$
\operatorname{erfcx}=\int_{x}^{\infty} e^{-u^{2}} d u
$$

$$
\left.\begin{array}{rl}
g_{1}^{\prime} & =\rho\left(3 f_{1}-s f_{1}^{\prime}\right), \\
2 \lambda_{1}\left(2 g_{1}-s g_{1}^{\prime}\right)+4 g_{0} & =2 \eta_{0} f_{0}^{\prime}+\eta_{0} \lambda_{2}\left(2 f_{1}^{\prime}-s f_{1}^{\prime \prime}\right), \\
2 \lambda_{1}\left(2 h_{1}-s h_{1}^{\prime}\right)+2 v_{1} g_{0} f_{0}^{\prime} & =\eta_{0} v_{2} f_{0}^{\prime 2}, \\
2 \lambda_{1}\left(2 k_{1}-s k_{1}^{\prime}\right)+2\left(v_{1}-2 \lambda_{1}\right) g_{0} f_{0}^{\prime} & =\eta_{0}\left(v_{2}-2 \lambda_{2}\right) f_{0}^{\prime 2} .
\end{array}\right\}
$$

The appropriate boundary conditions are

$$
f_{1}(0)=f_{1}(\infty)=0 \text {, }
$$

and $t g_{1}, t h_{1}, t k_{1} \rightarrow 0$ as $t \rightarrow 0$. The second of equations (12) may be written

$$
s^{3} \frac{d}{d s}\left(g_{1} / s^{2}\right)=\frac{a^{2} \rho}{2} s^{3} \frac{d}{d s}\left(f_{1}^{\prime} / s^{2}\right)+\frac{2 a \rho A\left(\lambda_{1}-\lambda_{2}\right)}{\lambda_{1} \lambda_{2}} \operatorname{erfc}(s / a)
$$

which is readily integrated to give $g_{1}$ in terms of $f_{1}$. Substituting for $g_{1}$ in the first of equations (12) gives an equation for $f_{1}$. The relevant solution of equations (12) is

$$
\begin{aligned}
& \begin{array}{r}
f_{1}(s)=\frac{A\left(\lambda_{1}-\lambda_{2}\right)}{\lambda_{1} \lambda_{2}}\left[\left(\frac{2 s^{3}}{a^{3}}+s / a\right) \operatorname{erfc}(s / a)-\frac{s^{2}}{a^{2}} e^{-s^{2} / a^{2}}\right] \\
g_{1}(s)=\frac{a \rho A\left(\lambda_{1}-\lambda_{2}\right)}{2 \lambda_{1} \lambda_{2}}\left[\left(\frac{2 s^{2}}{a^{2}}-1\right) \operatorname{erfc}(s / a)-s / a e^{-s^{2} / a^{2}}\right]
\end{array} \\
& \begin{aligned}
h_{1}(s)=k_{1}(s)=\frac{\rho A^{2}\left(\lambda_{1} v_{2}-\lambda_{2} v_{1}\right)}{\lambda_{1} \lambda_{2}}\left[\frac{s^{2}}{a^{2}} E i\left(\frac{2 s^{2}}{a^{2}}\right)\right. \\
\left.+\left(\frac{2 s^{2}}{a^{2}}+1\right)(\operatorname{erfc}(s / a))^{2}-\frac{2 s}{a} e^{-s^{2} / a^{2}} \operatorname{erfc}(s / a)\right], \\
\text { where } \quad E i(x)=\int_{x}^{\infty} \frac{e^{-u}}{u} d u .
\end{aligned}
\end{aligned}
$$

For solutions for large time let

$$
\begin{aligned}
v & =t^{\frac{1}{2}} F_{0}(s)+t^{-\frac{1}{2}} F_{1}(s)+\ldots, \\
p_{x y} & =G_{0}(s)+t^{-1} G_{1}(s)+\ldots \\
p_{x x} & =p_{z z}=H_{0}(s)+t^{-1} H_{1}(s)+\ldots, \\
p_{y y} & =K_{0}(s)+t^{-1} K_{1}(s)+\ldots
\end{aligned}
$$

From equations (4)-(8)

$$
\left.\begin{array}{rl}
G_{0}^{\prime} & =\rho\left(F_{0}-s F_{0}^{\prime}\right), \\
4 G_{0}-2 \lambda_{1} H_{0} F_{0}^{\prime}+\mu_{0} F_{0}^{\prime}\left(2 H_{0}+K_{0}\right) & =2 \eta_{0} F_{0}^{\prime}, \\
4 H_{0} & =F_{0}^{\prime}\left(v_{2} \eta_{0} F_{0}^{\prime}-2 v_{1} G_{0}\right), \\
4 K_{0} & =F_{0}^{\prime}\left\{\left(v_{2}-2 \lambda_{2}\right) \eta_{0} F_{0}^{\prime}-2\left(v_{1}-2 \lambda_{1}\right) G_{0}\right\} .
\end{array}\right\}
$$


The boundary conditions (9) give

$$
F_{0}(0)=A, \quad F_{0}(\infty)=0 .
$$

The second of equations (13) reduces to

$$
8 G_{0}=4 \eta_{0} F_{0}^{\prime}+F_{0}^{\prime 2}\left(\eta_{0} \sigma_{2} F_{0}^{\prime}-2 \sigma_{1} G_{0}\right)
$$

where

$$
\begin{aligned}
& \sigma_{1}=\lambda_{1} \mu_{0}+\left(\lambda_{1}-\frac{3 \mu_{0}}{2}\right) v_{1}, \\
& \sigma_{2}=\lambda_{2} \mu_{0}+\left(\lambda_{1}-\frac{3 \mu_{0}}{2}\right) v_{2}
\end{aligned}
$$

The first of equations (13) and equation (14) can be expressed in nondimensional form by the following substitutions,

$$
\begin{gathered}
s=v_{0}^{\frac{1}{2}} \vartheta, \quad \sigma_{1}=\alpha_{1} \lambda_{1}^{2}, \quad \sigma_{2}=\alpha_{2} \lambda_{1}^{2}, \\
F_{0}=A p, \quad G_{0}=A \rho v_{0}^{\frac{1}{2}} q .
\end{gathered}
$$

The equations become

$$
\begin{aligned}
q^{\prime} & =p-\vartheta p^{\prime} \\
8 q & =4 p^{\prime}+\tau^{2} p^{\prime 2}\left(\alpha_{2} p^{\prime}-2 \alpha_{1} q\right)
\end{aligned}
$$

where $\tau^{2}=\frac{A^{2} \lambda_{1}^{2}}{v_{0}}$. The boundary conditions for equations (15) are

$$
p(0)=1, \quad p(\infty)=0 .
$$

Since $\lambda_{1}$ is of order $10^{-1}$ sec. and $\eta_{0}$ is of order 10 pse or greater, $\tau^{2}$ can be assumed small provided $A$ is not too large. Writing

equations (15) give

$$
\begin{aligned}
& p=p_{0}+\tau^{2} p_{1}+\ldots, \\
& q=q_{0}+\tau^{2} q_{1}+\ldots,
\end{aligned}
$$

where

$$
\begin{aligned}
q_{0}^{\prime} & =p_{0}-\vartheta p_{0}^{\prime}, \\
2 q_{0} & =p_{0}^{\prime},
\end{aligned}
$$

$$
p_{0}(0)=1, \quad p_{0}(\infty)=0
$$

Thus

$$
\begin{aligned}
& p_{0}=e^{-\vartheta^{2}}-2 \vartheta \operatorname{erfc} \vartheta \\
& q_{0}=-\operatorname{erfc} \vartheta
\end{aligned}
$$

The equations for the coefficients of $\tau^{2}$ are

where

$$
\begin{aligned}
q_{1}^{\prime} & =p_{1}-\vartheta p_{1}^{\prime}, \\
2 q_{1} & =p_{1}^{\prime}+2\left(\alpha_{1}-\alpha_{2}\right)(\operatorname{erfc} \vartheta)^{3},
\end{aligned}
$$

$$
p_{1}(0)=p_{1}(\infty)=0
$$


Hence

$$
\begin{aligned}
& p_{1}=\left(\alpha_{1}-\alpha_{2}\right)\left[\frac{3(\pi-2)}{8}\left(2 \vartheta \operatorname{erfc} \vartheta-e^{-\vartheta^{2}}\right)\right.\left.-2 \vartheta(\operatorname{erfc} \vartheta)^{3}+\frac{3}{2} e^{-\vartheta^{2}}(\operatorname{erfc} \vartheta)^{2}+\frac{3}{2}^{\frac{3}{2}} \vartheta \operatorname{erfc}\left(3^{\frac{1}{2}} \vartheta\right)-\frac{3}{4} e^{-3 \vartheta^{2}}\right], \\
& q_{1}=\left(\alpha_{1}-\alpha_{2}\right)\left[\frac{3(\pi-2)}{8} \operatorname{erfc} \vartheta+\frac{3 \vartheta}{2} e^{-\vartheta^{2}}(\operatorname{erfc} \vartheta)^{2}-\frac{3}{2} e^{-2 \vartheta^{2}} \operatorname{erfc} \vartheta+\frac{3}{4}^{\frac{3}{2}} \operatorname{erfc}\left(3^{\frac{1}{2}} \vartheta\right)\right] .
\end{aligned}
$$

In the original notation,

$$
\begin{aligned}
& F_{0}(s)=A\left(e^{-\vartheta^{2}}-2 \vartheta \operatorname{erfc} \vartheta\right)+\frac{A^{3}\left(\sigma_{1}-\sigma_{2}\right)}{v_{0}}\left[\frac{3(\pi-2)}{8}\left(2 \vartheta \operatorname{erfc} \vartheta-e^{-\vartheta^{2}}\right)\right. \\
& \left.-2 \vartheta(\operatorname{erfc} \vartheta)^{3}+\frac{3}{2} e^{-\vartheta^{2}}(\operatorname{erfc} \vartheta)^{2}+\frac{3}{2}^{\frac{3}{2}} \vartheta \operatorname{erfc}\left(3^{\frac{1}{2}} \vartheta\right)-\frac{3}{4} e^{-3 \vartheta^{2}}\right]+0\left(\frac{A^{5} \lambda_{1}^{4}}{v_{0}^{2}}\right), \\
& G_{0}(s)=-\rho v_{0}^{\frac{1}{2}} A \operatorname{erfc} \vartheta+\frac{\rho A^{3}\left(\sigma_{1}-\sigma_{2}\right)}{v_{0}^{\frac{1}{2}}}\left[\frac{3(\pi-2)}{8} \operatorname{erfc} \vartheta\right.
\end{aligned}
$$$$
\left.+\frac{3}{2} \vartheta e^{-\vartheta^{2}}(\operatorname{erfc} \vartheta)^{2}-\frac{3}{2} e^{-2 \vartheta^{2}} \operatorname{erfc} \vartheta+\frac{3}{4}^{\frac{3}{2}} \operatorname{erfc}\left(3^{\frac{1}{2}} \vartheta\right)\right]+0\left(\frac{\rho A^{5} \lambda_{1}^{4}}{v_{0}^{\frac{3}{2}}}\right)
$$$$
H_{0}(s)=\rho A^{2}\left(v_{2}-v_{1}\right)(\operatorname{erfc} \vartheta)^{2}+0\left(\frac{\rho A^{4} \lambda_{1}^{3}}{v_{0}}\right)
$$

where

$$
\begin{gathered}
K_{0}(s)=\rho A^{2}\left(v_{2}-v_{1}+2\left(\lambda_{1}-\lambda_{2}\right)\right)(\operatorname{erfc} \vartheta)^{2}+0\left(\frac{\rho A^{4} \lambda_{1}^{3}}{v_{0}}\right), \\
\vartheta=s / v_{0}^{\frac{1}{2}}=x / 2\left(t v_{0}\right)^{\frac{1}{2}} .
\end{gathered}
$$

It is possible to proceed and evaluate $F_{1}, G_{1}, H_{1}, K_{1}$. However, these terms contain an undetermined constant due to the fact that a complementary function is zero at both boundaries. This is not surprising since the situation is similar to that in boundary layer problems where undetermined constants appear in the solution. The unknown constant in the present problem could be found by matching with a solution for smaller time.

An inspection of the first two terms of the solution for small time suggests that it is valid provided that $t$ is small compared with $\lambda_{1}$ and thus the solution is valid for only very small times. The series for large time would appear to be valid for time large compared with $\lambda_{1}$.

For a Newtonian fluid this problem has the exact solution

$$
\begin{aligned}
v & =A t^{\frac{1}{2}}\left(e^{-2^{2}}-2 \vartheta \operatorname{erfc} \vartheta\right), \\
p_{x y} & =-A \rho v_{0}^{\frac{1}{2}} \operatorname{erfc} \vartheta \\
p_{x x} & =p_{y y}=p_{z z}=0 .
\end{aligned}
$$

Initially both the velocity and the shear stress are significantly less for the non-Newtonian liquid than they are for the Newtonian liquid. The differences 
between the velocities and shear stresses decrease with time and at times large compared with the relaxation time the non-Newtonian values are smaller than the Newtonian values by a factor of order $\frac{A^{2} \lambda_{1}^{2}}{v_{0}}$ (since $\sigma_{1}>\sigma_{2}(\mathbf{1})$ ). In fig. 1 the velocity profiles are compared for small time and in fig. 2 the shear stresses are compared for small time. Initially the normal stresses are zero but at large time there is an extra tension along the streamlines.

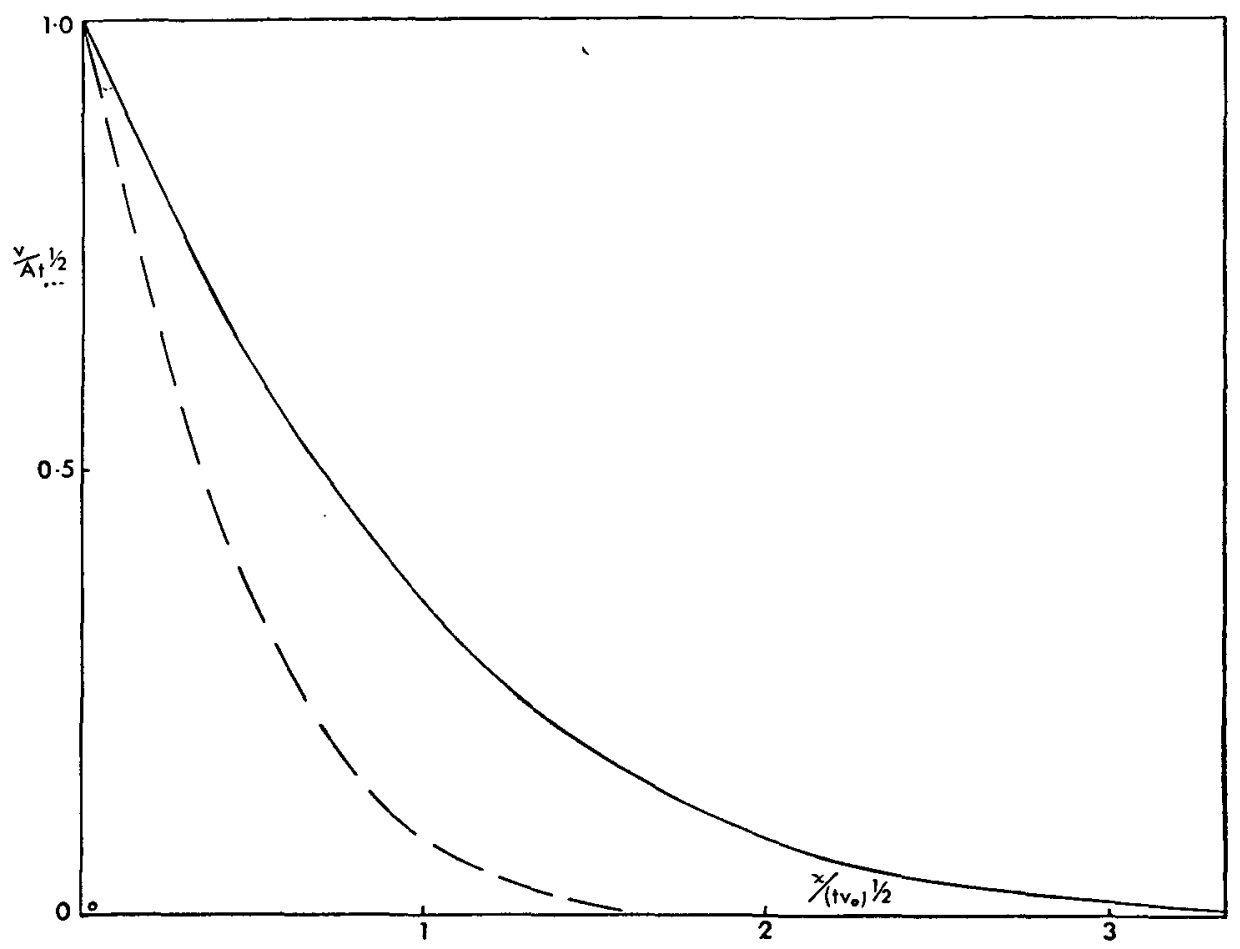

FIG. 1. - The velocity profiles compared at small time $\left(\lambda_{1}=4 \lambda_{2}\right)$. Newtonian. - - Non-Newtonian.

The drag $D$ on the plate is given by

$$
D=\left(\frac{\lambda_{2}}{\lambda_{1}}\right)^{\frac{1}{2}} D_{N}\left(1+\frac{\lambda_{1}-\lambda_{2}}{2 \lambda_{1} \lambda_{2}} t+\ldots\right)
$$

for small time, and for large time by

$$
D=D_{N}\left[1-\frac{3 A^{2}\left(\sigma_{1}-\sigma_{2}\right)}{4 v_{0}}\left(\pi / 2+3^{\frac{1}{2}}-3\right)+\ldots\right]
$$

where the Newtonian drag $D_{N}=\frac{A \rho\left(v_{0} \pi\right)^{\frac{1}{2}}}{2}$. In fig. 3 the drag $D$ is compared 


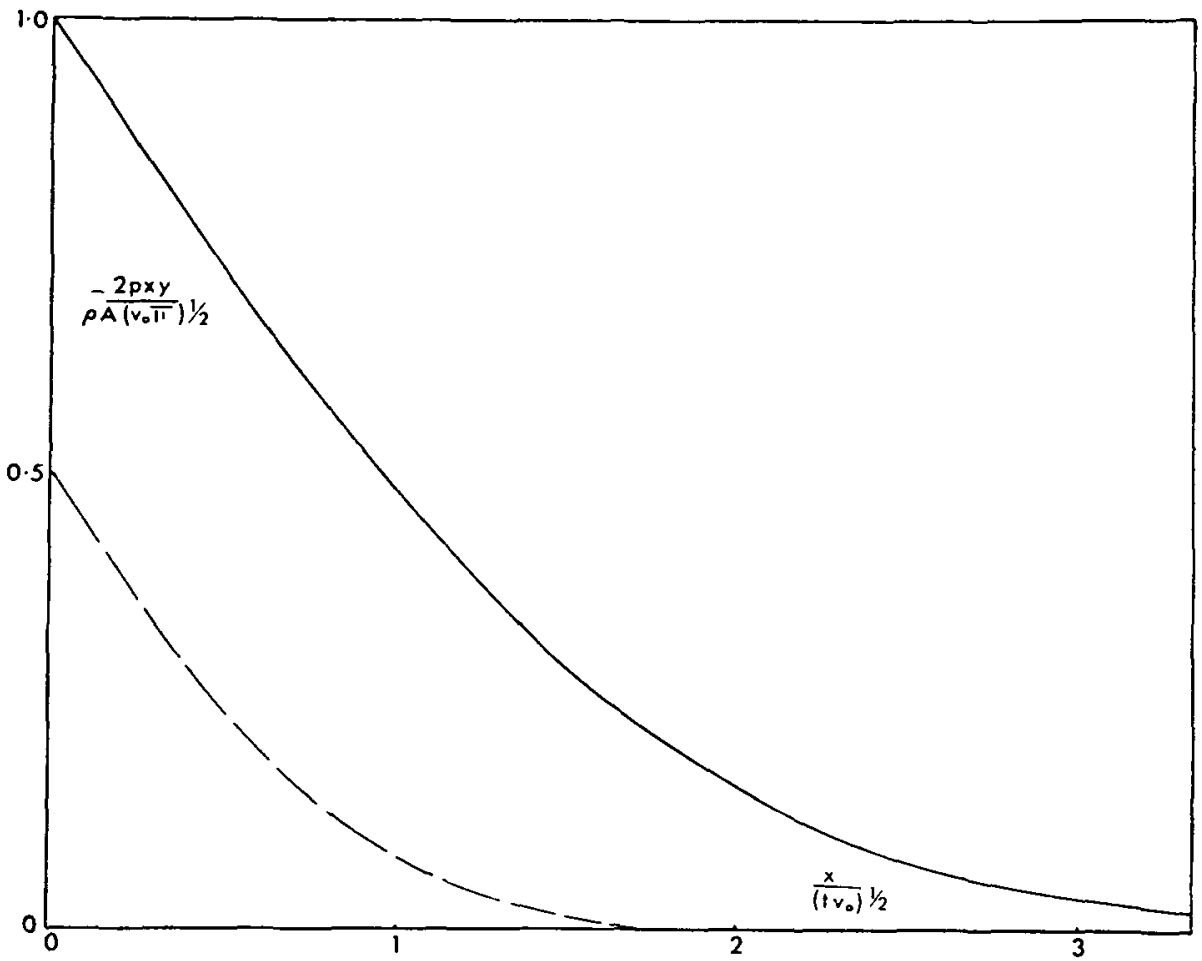

Fig. 2.-The shear stresses compared at small time $\left(\lambda_{1}=4 \lambda_{2}\right)$.

Newtonian. - - Non-Newtonian.

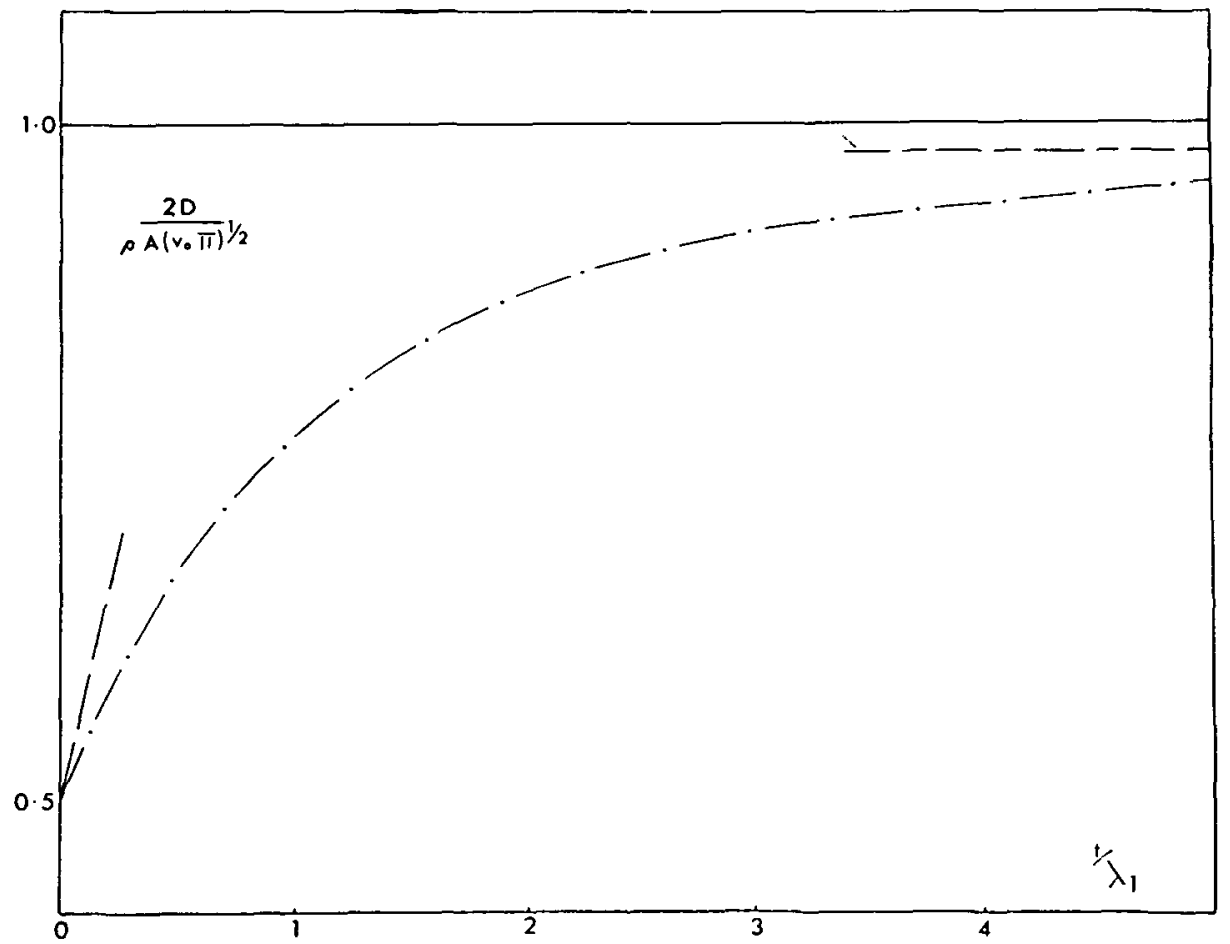
FIG. 3.-The drag against time $\left(\lambda_{1}=4 \lambda_{2}, \frac{A^{2}\left(\sigma_{1}-\sigma_{2}\right)}{\nu_{0}}=\frac{1}{10}\right)$. 
with this constant Newtonian drag. At first $D$ is much less than this value but increases rapidly with time. The asymptotic value for large time is a constant slightly smaller than the Newtonian value. From the first of equations (8) it can be seen that the normal component of stress on the plate is a constant pressure $P$.

\section{Approximate Solution by a Pohlhausen Method}

If the dimensionless parameter $A \lambda_{1} / v_{0}^{t}$ is small compared with unity, the non-linear terms in equations (4)-(7) can be neglected to give

$$
\left.\begin{array}{l}
p_{x x}+\lambda_{1} \frac{\partial}{\partial t} p_{x x}=0 \\
p_{y y}+\lambda_{1} \frac{\partial}{\partial t} p_{y y}=0 \\
p_{z z}+\lambda_{1} \frac{\partial}{\partial t} p_{z z}=0
\end{array}\right\}
$$

and

$$
p_{x y}+\lambda_{1} \frac{\partial}{\partial t} p_{x y}=\eta_{0}\left(\frac{\partial v}{\partial x}+\lambda_{2} \frac{\partial^{2} v}{\partial x \partial t}\right)
$$

Also

$$
\rho \frac{\partial v}{\partial t}=\frac{\partial p_{x y}}{\partial x}
$$

From the equations (16) and the boundary conditions (10)

$$
p_{x x}=p_{y y}=p_{z z}=0 \text {. }
$$

In this section an approximate solution of the equations (17), (18) is obtained in order to find an estimate of the drag on the plate at intermediate times.

Since the velocity and the shear stress are known at both large and small time from the solutions of the previous section, profiles are chosen for the velocity and the shear stress for all time such that they agree with the known values at small and large time. The profiles chosen are

$$
\begin{aligned}
v & =A t^{\frac{1}{2}}\left(e^{-\vartheta^{2} / \delta}-2 \vartheta / \delta^{\frac{1}{2}} \operatorname{erfc}\left(\vartheta / \delta^{\frac{1}{2}}\right)\right), \\
p_{x y} & =-\rho A\left(v_{0} \beta\right)^{\frac{1}{2}} \operatorname{erfc}\left(\vartheta / \beta^{\frac{1}{2}}\right),
\end{aligned}
$$

where $\vartheta=s / v_{0}^{\frac{1}{2}}=x / 2\left(t v_{0}\right)^{\frac{1}{2}}$ and $\delta$ and $\beta$ are functions of time. If $\delta$ and $\beta$ are both equal to unity, the profiles are those obtained in the previous section for large time if terms of order $A^{2} \hat{\lambda}_{1}^{2} / v_{0}$ are neglected. If $\delta$ and $\beta$ are both equal to $\lambda_{2} / \lambda_{1}$, the profiles are those obtained in the previous section for small time. Thus the boundary conditions for $\delta$ and $\beta$ are

$$
\begin{array}{ll}
\delta=\beta=\lambda_{2} / \lambda_{1}, & \text { when } t=0, \\
\delta=\beta=1, & \text { when } t=\infty .
\end{array}
$$


The functions $\delta$ and $\beta$ are determined from the equations obtained by integrating the equations (17) and (18) with respect to $x$ from 0 to $\infty$,

$$
\int_{0}^{\infty} p_{x y} d x+\lambda_{1} \frac{d}{d t} \int_{0}^{\infty} p_{x y} d x=-\eta_{0} v_{x=0}-\eta_{0} \lambda_{2} \frac{d}{d t}\left(v_{x=0}\right),
$$

and

$$
\rho \frac{d}{d t} \int_{0}^{\infty} v d x=-\left(p_{x y}\right)_{x}=0
$$

From equation (19),

$$
t^{\frac{1}{2}} \beta+\lambda_{1} \frac{d}{d t}\left(t^{\frac{1}{2}} \beta\right)=t^{\frac{1}{2}}+\lambda_{2} / 2 t^{\frac{1}{2}}
$$

and hence

$$
\beta=1-\left(1-\frac{\lambda_{2}}{\lambda_{1}}\right) \frac{\lambda_{1}^{\frac{t}{1}}}{t^{\frac{1}{2}}} e^{-t / \lambda_{1}} \int_{0}^{t^{1 / 2 / \lambda_{1}^{1 / 2}}} e^{u^{2}} d u
$$

From equation (20),

$$
\frac{d}{d t}\left(t \delta^{\frac{1}{2}}\right)=\beta^{\frac{1}{2}}
$$

From which $\delta$ can be found;

$$
\delta=\left(\frac{1}{t} \int_{0}^{t} \beta^{\frac{1}{2}} d u\right)^{2} .
$$

The drag on the plate, $D$, is given by

$$
D=\frac{\rho A\left(v_{0} \pi \beta\right)^{\frac{1}{2}}}{2} \text {. }
$$

In fig. $3, D$ is shown as a function of time when $\lambda_{1}$ is equal to $4 \lambda_{2}$. The drag increases quite quickly at first but approaches its asymptote rather slowly.

By comparing the quantity $\left(\frac{d D}{d t}\right)_{t=0}$ with its value from the previous section an indication of the accuracy of the Pohlhausen solution is obtained. For the Pohlhausen solution

$$
\left(\frac{d D}{d t}\right)_{t=0}=\frac{\rho\left(v_{0} \pi\right)^{\frac{1}{2}} A\left(\lambda_{1}-\lambda_{2}\right)}{6 \lambda_{1}^{2}}\left(\frac{\lambda_{1}}{\lambda_{2}}\right)^{\frac{1}{2}}
$$

and for the series solution

$$
\left(\frac{d D}{d t}\right)_{t=0}=\frac{\rho\left(v_{0} \pi\right)^{\frac{1}{2}} A\left(\lambda_{1}-\lambda_{2}\right)}{4 \lambda_{1}^{2}}\left(\frac{\lambda_{1}}{\lambda_{2}}\right)^{\frac{1}{2}}
$$

Thus this quantity is too small by 30 per cent. in the Pohlhausen solution and this suggests that the drag would tend to its asymptotic value more rapidly than indicated by this approximate solution. 


\section{Acknowledgment}

Part of this research was supported by the U.S. Air Force under Contract AF 49(638)-708, monitored by the Air Force Office of Scientific Research of the Air Research and Development Command.

\section{REFERENCES}

(1) J. G. Oldroyd, P.R.S.A., 245 (1958), 278.

(2) J. G. OLDROYD, P.R.S.A., 200 (1950), 523.

(3) F. M. LesLIE, Q.J.M.A.M., 14 (1961), 36.

(4) J. G. OldRoYd, Q.J.M.A.M., 4 (1951), 271.

(5) B. A. Toms and D. J. Strawbridge, Trans. Farad. Soc., 49 (1953), 1225.

DePaRTMENT OF MATHEMatics

KING'S COLLEGE

NEWCASTLE-UPON-TYNE 1 